outcomes were adequate although $11 / 25$ were lost to followup (did not attend their end of treatment consultation) including 5 prisoners (released or transferred).

Conclusions Treating TB in prisoners and homeless persons continues to be a challenge, even when DOT is undertaken. Tablet counting can help identify potential non-compliance in persons without obvious risk factors. In our experience, tablet counting is an effective and relatively cheap objective adjunct in the assessment of ATT compliance.

\section{P168 SHOULD WE CONTINUE SCREENING HOUSEHOLD CONTACTS OF ALL INDEX CASES WITH TB IRRESPECTIVE OF INFECTIVITY?- AN ANALYSIS OF CONTACT SCREENING YIELDS STRATIFIED ACCORDING TO INDEX SITE OF DISEASE AND SMEAR STATUS}

R Enuechie, M Kanu, A Amoah, J Marshall, M Ogundengbe, F Ogunrin, LV Baker. University Hospital Lewisham, London, UK

\subsection{6/thoraxjnl-2017-210983.310}

Aim NICE Guidance (2016) recommends that TB contact screening is only carried out on close contacts (household and workplace/school contacts) of patients with infectious tuberculosis ie pulmonary tuberculosis (AFB smear positive) and laryngeal tuberculosis. However previous guidance recommended screening all household contacts of any index with TB irrespective of infectious status. The aim of this study was to look at the yields of contact screening amongst 3 groups of index cases- infectious smear positive pulmonary TB, smear negative pulmonary $\mathrm{TB}$ and extrapulmonary TB.

Method We analysed our records for contact screening of index cases with tuberculosis notified between January 2011 and May 2016. Index cases were divided into pulmonary smear positive, pulmonary smear negative and extrapulmonary. Contacts were divided into close, casual and workplace. The screening yields for each population were compared.

Results Between 1 st January 2011 and 31 st May 20161887 contacts of 408 notified index cases with TB were screened; 1109 were screened as contacts of smear positive pulmonary $\mathrm{TB}, 176$ contacts of smear negative pulmonary TB, 506 contacts of extrapulmonary $\mathrm{TB}$, the remainder the index site of disease was not specified. CXR screening was performed on the 510 contacts over the age of 35 (2011 guidelines). Patients 35 and under had 2 step immunological assessment with Mantoux and IGRA. There was a strong correlation between size of Mantoux response and IGRA positivity; 6\% of Mantoux $<6 \mathrm{~mm}, 23 \%$ Mantoux 6-10 mm, 40\% Mantoux 11-15 mm, 55\% Mantoux 15-20 mm, $84 \%$ Mantoux $\geq 25 \mathrm{~mm}$. 604 contacts of index cases with AFB smear positive sputum were assessed immunologically - 123 (20.3\%) were positive, 136 contacts of AFB smear negative pulmonary TB were assessed - $19(10.5 \%)$ were positive, and 383 contacts of extrapulmonary TB were assessed - 42 (11\%) were positive. 26 of 239 (11\%) workplace/school contacts of infectious TB were positive, compared to $21.5 \%$ of close/casual contacts.

Conclusions Although contact screening yields for index cases with smear positive pulmonary $\mathrm{TB}$ are high, the Results for extrapulmonary and smear negative pulmonary $\mathrm{TB}$ are not insubstantial. Our data would suggest that we should continue screening close contacts of all TB index cases irrespective of infectious status.

\section{P169 \\ LATENT TUBERCULOSIS INFECTION SCREENING OF ADULT CLOSE CONTACTS IN LONDON: A COST-UTILITY ANALYSIS}

${ }^{1} \mathrm{M}$ Hayama, ${ }^{1} \mathrm{~N}$ Green, ${ }^{2} \mathrm{SL}$ Seneviratne, ${ }^{3} \mathrm{M}$ O'Donoghue, ${ }^{4} \mathrm{~N}$ Drey, ${ }^{3} \mathrm{OM}$ Kon. ${ }^{1} \mathrm{NIHR}$ Health Protection Research Unit in Modelling Methodology and MRC Centre for Outbreak Analysis and Modelling, Imperial College London School of Public Health, London, UK; ${ }^{2}$ Clinical Immunology, Royal Free Hospital, London, UK; ${ }^{3}$ Chest and Allergy Department, St. Mary's Hospital, Imperial College NHS Trust, London, UK; ${ }^{4}$ School of Health Sciences, City, University of London, London, UK

\subsection{6/thoraxjnl-2017-210983.311}

Background The National Institute for Health and Care Excellence (NICE) guidelines in 2016 recommend tuberculin skin test (TST) at a $5 \mathrm{~mm}$ induration size cut-off for latent tuberculosis infection (LTBI) screening of adult close contacts of active tuberculosis (TB) cases. An alternative would be to use an interferon-gamma release assay (IGRA) which has a higher specificity, such as the QuantiFERON-TB Gold in Tube (QFTGIT) or T-SPOT.TB (T-SPOT). We aimed to evaluate the costeffectiveness of the screening and treatment of LTBI in adult close contacts with various combinations of these tests in a representative London cohort.

Methods Clinical data of adult close contacts of pulmonary TB cases who were recommended to receive TST and IGRA in a TB clinic in London between 2008 and 2010 were retrospectively reviewed. A Markov decision analytic model, using an NHS perspective and lifetime horizon, was used to compare costs and quality-adjusted life-years (QALYs) associated with 7 screening strategies followed by chemoprophylaxis: TST alone, IGRA (QFT-GIT or T-SPOT) alone, TST positive followed by IGRA, and TST negative followed by IGRA. Future costs and QALYs were discounted at 3.5\% per year.

Results 381 asymptomatic close contacts aged 18 to 65 years were included in this study. The mean age was 35.2 years and the majority $(75.3 \%)$ were BCG vaccinated. In the base-case analysis, QFT-GIT was the most cost-effective strategy with $£ 6876$ per QALY gained, compared to TST positive followed by QFT-GIT strategy. QFT-GIT alone averted 1.6 TB cases per 1000 contacts compared to TST positive followed by QFTGIT.

Conclusion Of the considered testing strategies, the QFT-GIT alone is preferable for LTBI screening in adult close contacts of pulmonary $\mathrm{TB}$ cases in London.

\section{P170 NEW ENTRANT LATENT TUBERCULOSIS SCREENING IN THE UK: SHOULD THE SEARCH BE WIDENED?}

MS Rana, S Sandhu, M Silka, SM Menzies. Wexham Park Hospital, Slough, UK

\subsection{6/thoraxjnl-2017-210983.312}

Introduction Most Tuberculosis (TB) cases in England are the result of reactivation of latent TB infection (LTBI) in foreignborn immigrants. The Collaborative TB Strategy recommends LTBI screening in New Entrants, who have entered the UK in the previous 5 years, are aged 16-35 and from countries with a TB incidence of $\geq 150$ cases/100,000 population. ${ }^{1}$ This is seen as cost-effective, ${ }^{2}$ but in contrast to National Institute for Health and Care Excellence (NICE) guidance, which recommends screening those aged 0-65 from countries with a lower TB incidence of $\geq 40$ cases/100,000). This study evaluated how the two screening programmes compared. 
Methods Patients screened in 2016 by our Secondary Care service using NICE-recommendations were included. Those aged 16-65 had an Interferon Gamma Test (QuantiFERON) and those aged 0-16 a Mantoux Test (reported as positive if $\geq 5 \mathrm{~mm}$ ). Results were then stratified by the TB incidence in their country of birth and by age.

Results 345 patients were offered screening, and 235 patients attended (68\%). 44 patients (19\%) were found to have LTBI and none had active TB. The Results are displayed in Table 1. 120 patients $(51 \%)$ were in the Strategy-recommended group, which had the lowest LTBI-positivity rate (10\%). Restricting screening to just this group would have resulted in 32 of the 44 LTBI cases (72\%) being 'missed'. The LTBI-positivity rate was high in the younger and older age groups from the $\geq 150$ cases/100,000 countries ( $25 \%$ and $33 \%$ respectively), and $1 / 3$ of those screened in the age 16-35 group from the 40-150 cases/100,000 countries tested positive, the majority being Romanian.

Conclusions The LTBI rate in New Entrants is high in groups not currently widely screened. Broadening the programme to include patients from a wider age range and from countries with a TB incidence of $\geq 40 / 100,000$ would achieve higher LTBI detection and aid National TB control.

\section{REFERENCES}

1. Collaborative TB Strategy for England 2015-2020: PHE 2015, January.

2. Pareek M, et al. The Lancet Infectious Disease 2011;11:435-444.

Abstract P170 Table 1 LTBI screening results stratified by TB incidence in the country of birth and by age range (shaded cells indicate the Strategy-recommended screening group results)

\begin{tabular}{|c|c|c|}
\hline $\begin{array}{l}\text { Age range } \\
\text { ( } n=235 \text { patients) }\end{array}$ & $\begin{array}{c}\text { TB rate } 40-150 / 100,00 \\
\text { ( } n=72 \text { patients) }\end{array}$ & $\begin{array}{c}\text { TB rate } \geq 150 / 100,000 \\
\text { ( } n=163 \text { patients) }\end{array}$ \\
\hline \multirow[t]{2}{*}{0.15 years } & Screened = 1 & Screened $=16$ \\
\hline & Positive $=1(100 \%)$ & Positive $=4(25 \%)$ \\
\hline \multirow[t]{2}{*}{$16-35$ years } & Screened = 52 & Screened $=120$ \\
\hline & Positive $=15(29 \%)$ & Positive $=12(10 \%)$ \\
\hline \multirow[t]{2}{*}{$36-65$ years } & Screened $=19$ & Screened $=27$ \\
\hline & Positive $=3(16 \%)$ & Positive $=9(33 \%)$ \\
\hline
\end{tabular}

\section{Pulmonary vascular disease: monitoring and managing}

\section{P171 ARE THE EUROPEAN SOCIETY OF CARDIOLOGY PULMONARY HYPERTENSION GUIDELINE RISK ASSESSMENT CRITERIA ASSOCIATED WITH 12-MONTH MORTALITY?}

${ }^{1} \mathrm{C}$ Sharp, ${ }^{1} \mathrm{~A}$ Grove, ${ }^{1} \mathrm{D}$ Augustine, ${ }^{1} \mathrm{~K}$ Carson, ${ }^{1} \mathrm{~J}$ Easaw, ${ }^{1} \mathrm{~T}$ Hall, ${ }^{1} \mathrm{~B}$ Hudson, ${ }^{1} \mathrm{G}$ Robinson, ${ }^{2} \mathrm{G}$ Coghlan, ${ }^{1} \mathrm{R}$ Mackenzie-Ross, 'I Suntharalingam. 'Royal United Hospitals Bath NHS Foundation Trust, Bath, UK, ${ }^{2}$ Royal Free London NHS Foundation Trust, London, UK

\subsection{6/thoraxjn-2017-210983.313}

Background The European Society of Cardiology (ESC) guidelines for management of pulmonary hypertension (PAH) advocate comprehensive assessment of patients to determine prognosis and to guide treatment decisions, using a set of risk assessment criteria based on expert advice. These criteria are coded Red (high), Amber (medium) and Green (low). It is unclear whether these criteria are associated with short term survival.

Aim To determine whether red/amber/green risk status according to ESC guidelines is associated with 12 month mortality.

Methods This was a "snapshot" observational study using routinely collected clinical data for patients eligible for targeted drug treatment at a regional centre, under shared care with a national centre. All data available at the latest visit within the study period were collated, including demographics, echocardiogram and right heart catheterisation data. Data are reported as mean $/$ median $/$ count $/ \%$. Characteristics of deceased and surviving patients were compared using Mann-Witney Utest. Association with 12 month mortality was assessed using Receiver Operator Characteristics (ROC) curve analysis.

Results Routinely collected clinic data were available for 104 patients, echocardiograms for 88 and right heart catheter data for 68 . 25\% were male, mean age 68.2 years. $45.2 \%$ had connective tissue disease-associated $\mathrm{PAH}, 32.7 \%$ inoperable chronic thromboembolic PH, 18.3\% Idiopathic PAH. 101 were on treatment, of which $35.6 \%$ were on monotherapy, $51.0 \%$ on dual oral therapy, $9.6 \%$ on intravenous treatments. Baseline data are shown in the table. $25 \%$ had one red criterion, $14.4 \%$ had two and $8.6 \%$ had three or more. 19 patients died in the 12 month follow-up period, 6 of whom had no red criteria. Deceased patients were older $(p=0.015)$ and had shorter walking distance $(\mathrm{p}=0.003)$. Risk criteria were worse for symptom progression, WHO functional class, walking distance and for the overall number of red criteria. ROC-curve analysis showed that symptom progression (c-statistic $0.695, \mathrm{p}=0.048)$, walking distance $(0.748, \mathrm{p}=0.012)$ and the overall number of red flags $(0.710, p=0.033)$ were the only elements associated with 12 month mortality.

Conclusions The ESC risk assessment criteria are associated with 12 month mortality in this cohort when all criteria are collated. Further work in a large cohort is needed to confirm the clinical utility of these criteria.

\begin{tabular}{lll} 
Abstract P171 Table 1 & & \\
\hline & Median/\% & Red flags, $\mathrm{n}(\%)$ \\
\hline Symptom progression & N/A & $11(10.6)$ \\
NT-proBNP (ng/L) & 60.5 & $2(1.9)$ \\
WHO functional class & N/A & $13(12.5)$ \\
6 min walk distance (m) & 295 & $28(28)$ \\
Echo RA area (cm2) & 17.5 & $5(7.4)$ \\
Right heart catheter (any criterion) & N/A & $13(19.1)$ \\
\hline
\end{tabular}

\section{P172 PRE-OPERATIVE INSIGHTS FROM CARDIOPULMONARY EXERCISE TESTING IN PATIENTS WITH PULMONARY ARTERIOVENOUS MALFORMATIONS}

${ }^{1} S$ Thurairatnam, ${ }^{2} \mathrm{~V}$ Santhirapala, ${ }^{2} \mathrm{~T}$ Hall, ${ }^{3} \mathrm{HC}$ Tighe, ${ }^{3} \mathrm{~J}$ Perks, ${ }^{3} \mathrm{JE}$ Jackson, ${ }^{4} \mathrm{LS}$ Howard ${ }^{4} \mathrm{CL}$ Shovlin. ${ }^{1}$ Hull York Medical School, York, UK; ${ }^{2}$ Imperial College School of Medicine, London, UK; ${ }^{3}$ Imperial College Healthcare NHS Trust, London, UK; ${ }^{4}$ Imperial College London and Imperial College Healthcare NHS Trust, London, UK

10.1136/thoraxjnl-2017-210983.314 